\title{
Zusammenfassung und Schlussbemerkungen
}

Interessanter und überraschender als die Schlussfolgerungen dieser Arbeit sind, so meine ich, die gedanklichen Bahnen, die es dazu einzuschlagen galt, und die sich im Prozess chronologischer Darstellung abzeichnenden Verbindungslinien zwischen Texten unterschiedlicher Provenienz. Wie sich motivische und literarische Muster fortschreiben, verdichten, ausdünnen, überlagern, wie sie rezipiert, tradiert und adaptiert werden, habe ich versucht, anhand bekannter und weniger bekannter Quellen aufzuzeigen, wobei neben der interpretatorischen Analyse die soziohistorische, mentalitätsgeschichtliche und biographische Verortung der Texte einen umfassenden Blick auf das literarische Geschehen ermöglichen sollte.

Was wissen wir also am Ende über die »Landjuden« in der deutschen und deutschjüdischen Literatur vor 1918? Die bereits eingangs geäußerte Annahme, mit dem Auftreten dörflicher Juden als distinkter Kategorie sei erst zu rechnen, sobald diese nicht mehr das Gros der jüdischen Bevölkerung stellten und sich klar von ihren städtischen Glaubensbrüdern unterschieden, erweist sich selbstverständlich als berechtigt - und gleichzeitig als Tautologie: Denn wie eine abgehoben "gebildete« oder bürgerliche Perspektive der Erzählliteratur qua Herkunft inhärent ist, verrät der generalisierende Begriff »Landjude« eine ganz ähnliche Geisteshaltung. Der Gegensatz zweier - zumindest in der Vorstellung - intellektuell unterschiedlich gearteter Sphären, sittlicher Niveaus, wirtschaftlicher Ordnungen und ästhetischer Maßstäbe mit Namen "Stadt» und »Land« gehört zu den gedanklichen Voraussetzungen der neueren europäischen Literatur überhaupt. Diese grundlegende Annahme historisch zu präzisieren, war eine der Herausforderungen, der sich diese Arbeit zu stellen hatte; eine zweite, alternative Herangehensweisen an die Thematik zu erwägen und praktisch anzuwenden.

Historisch lässt sich eine interne Differenzierung des aschkenasischen Judentums anhand von Infrastruktur und Bildungsstand bereits in mittelalterlichen und frühneuzeitlichen Quellen nachweisen: Während eine »Kehilla« ein funktionierendes Gemeinwesen mit geordneten Gottesdiensten und Zugang zu rabbinischen Autoritäten darstellt, bezeichnet »jischuw« eine (meist dörfliche) Siedlung mit einer nur wenige Köpfe zählenden Judenschaft. Ohne Anschluss an größere Gemeinden verfügten dessen Bewohner oft nur über rudimentäre religionsgesetzliche Kenntnisse. Von »am ha'aretz« ist dann die Rede, von religiös Ungebildeten, auf deren Praxis kein Verlass sei, weil Willkür und Zuwiderhandeln Tür und Tor offenstehe. 
Die Zersplitterung in kleine und kleinste, oft kaum funktionstüchtige Gemeinwesen sowie das Phänomen der Vereinzelung sind für deutsche Verhältnisse nach den spätmittelalterlichen Vertreibungen bezeichnend, bestehen aber noch über die Zeit des Dreißigjährigen Krieges hinaus, als sich jüdisches Leben auf deutschem Boden abseits der einstigen Zentren (mit wenigen Ausnahmen wie Frankfurt) in überwiegend agrarisch geprägten Ortschaften abspielte.

Wie die für das deutsche Judentum später als charakteristisch empfundene Annahme bürgerlicher Formen und klassisch-humanistischer Bildungsinhalte hatte auch jene Epoche das Gepräge deutschen Judentums maßgeblich mitbestimmt. Denn die Verarmung weiter Teile der Bevölkerung im 17. und 18. Jahrhundert traf die restriktiven Sonderbestimmungen unterworfenen Juden besonders hart. Der Typus des »armen Juden«, der kaum sein Auslangen in Handel oder Bettel fand, war den Aufklärern und Emanzipationsbefürwortern durchaus ein Begriff, der mit einer ländlichen Daseinsform assoziiert war. In der schönen Literatur spielt dieser Typus eine untergeordnete Rolle (Miller, Siegwart) und stellt eine Spielart des »Handelsjuden«, aber auch des abgesondert nach den Vorschriften seiner Religion lebenden Juden dar, wie Judenfiguren insgesamt stereotyp konstruiert und auf die Rolle des gelegentlich zaubermächtigen Christusverleugners (Sage, Legende), des habgierigen Wucherers oder Kleinhändlers (Drama, Schwank) oder des »edlen Juden« (zunächst orientalischer Herkunft) beschränkt blieben.

Eine Abweichung vom Schema findet sich in der Schwankliteratur der Frühen Neuzeit (Heinrich Bebel), wenn jüdische Protagonistinnen und Protagonisten eines denkwürdigen, zum Lachen anreizenden Ausspruchs wegen erinnert werden, nicht unbedingt namentlich, so doch unter Angabe ihres Herkunfts- oder Wohnorts. Die persönliche Begegnung oder Kunde aus erster Hand war mit einem Zugeständnis an Individualität verbunden, das dem antijüdisch-christlichen Vorurteil zuwiderlief. In Schwänken und Anekdoten figurierten eben nicht ausschließlich die tölpelhaften oder geldgierigen Juden aus »entlegenen« Städten, die für ihre große jüdische Gemeinde bekannt waren (Prag, Frankfurt, etc.), sondern durchaus schlagfertige Hechinger oder Dillinger Juden. Dass diese Wahrnehmung auf die nachbarschaftliche Nähe in kleinen Ortschaften und eine entspanntere, eben nicht (ausschließlich) ressentimentgeladene Beziehung abstellt oder angetan war, diese zu befördern, lässt sich anhand des vorliegenden Quellenmaterials nicht entscheiden, liegt aber zumindest im Bereich des Vorstellbaren.

Das Bild des Juden aus der unmittelbaren Umgebung zu evozieren, in Gestalt des ortsansässigen oder -bekannten Händlers und Handelsvermittlers, gelang Johann Peter Hebel in seinen Kalendergeschichten, die thematisch an die Tradition 
des Judenschwanks anknüpfen, jedoch diese im Sinn der emanzipationsbefürwortenden Volksaufklärung modifizieren bzw. revidieren. Entscheidend dafür war nicht nur die Lokalisierung mehrerer Episoden im badischen bzw. alemannischen Raum, sondern auch der beabsichtigte Realitätseffekt in Sprache und Gestus, der ein »Wiedererkennen« ermöglichen sollte. Anstelle mit »edlen Juden« an ein humanistisches Sentiment zu appellieren, entwirft Hebel gewitzte, lebenskluge und weise Juden, die sich in jeder Lebenslage $\mathrm{zu}$ helfen wissen und von denen man mit Fug und Recht eine der aufklärerischsten aller Tugenden lernen könne: den Verstandesgebrauch. Den von Vorwitz korrumpierten Schwankjuden indes gewinnt Hebel allgemein-menschliche Seiten ab. Als Vertreter eines Volkes mit tragischem Schicksal, so der Tenor, bedürfen sie der moralischen wie politischen Unterstützung zur Eingliederung in die bürgerliche Gesellschaft und tragen durchaus Züge einer weiteren, bereits bekannten Figur: des armen, geplagten oder unterdrückten Juden.

Das uneingeschränkte Erbe des denunziatorischen Judenschwanks trat im ersten Drittel des 19. Jahrhunderts die sog. Emanzipationssatire an, die »den Landjuden« im preußischen Osten Deutschlands (Schlesien) schon früh zum Gegenbild des bildungsbeflissenen, städtischen Juden mit unterstellt ästhetischen Allüren und gleichzeitig zum Inbegriff des Juden schlechthin stilisierte: primitiv, triebgesteuert, unansehnlich (Fliegende Wische aus dem Ränzel eines zottigen Landjuden). Ein gutes Jahrzehnt später vernahm man ähnliche Töne aus Franken, wo die pseudonyme Autorgestalt des Itzig Feitel Stern schließlich zum fiktiven Herausgeber einer äußerst erfolgreichen antijüdischen Schriftenreihe avancierte und von Band zu Band deutlicher pseudobiographische Konturen gewann: nämlich die eines im dörflichen Bayern beheimateten Händlers, der sich zum Dichter berufen fühlt. Anders als ihr preußisches Pendant verfolgte die fränkische Satireschrift eine zweifache Absicht: nämlich sowohl die »neumodischen« als auch die »altmodischen« Juden moralisch zu diskreditieren. Denunziert wurde neben den Eingliederungs- und Bildungsabsichten auch die jüdische Religion als mittelalterlich-abergläubischer Obskurantismus, der sich ebenso materiell-pekuniären Interessen beuge, wie die Akkulturationsbestrebungen lediglich eine Gewinnmaximierung bezweckten.

Die bereits früh implizierte Differenzierung in primitive Dorfjuden und ambitionierte, Weltmännischkeit vorgaukelnde Bildungsbürger präsentiert sich erst in den 1850er Jahren als innerjüdische Hierarchisierung im Gefolge der Emanzipation, als in einer späten Itzig-Feitel-Stern-Schrift erstmals Anekdoten über einfältige Dorfjuden und etablierte Stadtjuden auftauchen. Der Wechsel von Außensicht zu einer Innenwahrnehmung, die den Gegensatz von Stadt und Land auf jüdische Verhältnisse im Süden und Westen Deutschlands überträgt, vollzieht 
sich zeitgleich in der volksnahen Erzählliteratur: In den 50er und 60er Jahren des 19. Jahrhunderts wird »der Dorfjude« regelrecht zum Typus innerhalb der deutsch-jüdischen Literatur ausgebaut (Alexander Weill, Simon Krämer), während zuvor die gelegentlichen Juden auf den Dörfern im Großen und Ganzen dem literarischen Vorbild des Hausierers nachempfunden gewesen waren, mochte man ihnen auch die eine oder andere »ländliche« Eigenheit unterstellt haben. Denn in den Schwarzwälder Dorfgeschichten Auerbachs und den frühen Elsässischen Sittengemälden Alexander Weills etwa werden die jüdischen Dorfgeher vornehmlich auf ihre jüdische Qualität schlechthin befragt, die sich an Verhalten und Lebenswandel der christlichen Mehrheitsgesellschaft zu messen hatte. Als Nebenfiguren waren sie dazu bestimmt, ein überragendes menschliches Mitgefühl und große Weltklugheit bis hin zur prophetischen Inspiration zu demonstrieren - »edle« Juden auf dem Dorf, gewiss, aber doch keine »Dorfjuden«.

Historisch korrespondiert die literarische Formierung des »Dorfjuden« mit dem beginnenden demographischen Verfall der dörflichen Kehillot. Hatte die Abwanderung schon Jahrzehnte zuvor eingesetzt, intensivierte sich diese ab Jahrhundertmitte (besonders nach Aufhebung des bayrischen Matrikelgesetzes 1861), sodass die Zahl der jüdischen Einwohner kontinuierlich sank. Gattungsmäßig verwandt war der »Dorfjude« dem »Ghettojuden« Kompertschen Zuschnitts, weil er in seiner Gegenbildlichkeit zu einem bürgerlich-säkularisierten Judentum eine ähnliche Funktion besaß: des traditionellen Gegenpols und Geschöpfs einer der Vergangenheit anheimgegebenen Ordnung.

Hervorstechende Merkmale des »Dorfjuden« waren sein Traditionalismus, seine Authentizität oder Primitivität, welche, je nach Sichtweise, positiv oder negativ konnotiert sein konnten, allenfalls aber ambivalent blieben: die ländlichen Juden, so eine der gedanklichen Grundvoraussetzungen des Typus, hatten sich eine Ursprünglichkeit bewahrt, die in einem Mangel an Umgangsformen und einer nüchternen Betrachtung der Realität zum Ausdruck kam. Geprägt hatte den »Dorfjuden« der Kampf um Subsistenz, hatte ihn emotional abgestumpft, ihm aber auch ein natürliches Gefühl für wahre Not erhalten (Simon Krämer, Salomon Hermann Mosenthal, Arthur Kahn). Beirren ließ sich der Dorfjude nicht. Sein Potential zum Schrullenhaften liegt in einer Spontaneität begründet, die der Zivilisationsprozess nicht einzuholen vermocht hat.

Die Anfänge des zeitgenössischen Materialismus führt Weill in seinen späten jüdischen Dorfgeschichten (1850er) ebenso auf ländlichen Erwerbszwang und Dünkel zurück wie auf eine tiefempfundene, biblisch fundierte Weltfrömmigkeit, aus der sich das Judentum zu erneuern habe. Die schlichte, auf das Notwendigste 
beschränkte Lebensweise konnte, wie bei Arthur Kahn und Pinchas Kohn, zur Bedingung spiritueller Reinheit werden.

Einmal abgesehen von der ästhetischen (und sentimentalen) Erbauung, welche die jüdischen Dorfgeschichten der zweiten Hälfte des 19. Jahrhunderts zu bieten hatten, hielten sie Argumente für eine Bewertung der schwindenden Kleingemeinden auf dem Land bereit: Besonders in den Schriften der reformnahen und liberalen Schriftsteller steht die dörfliche Kehilla als Sinnbild einer kollektiven oder individuellen Herkunft, von der eine Einbahnstraße ins städtische, die alte Tradition reflektierende Leben führt. Bei aller Nostalgie überwog die Erleichterung, weil man Integration, Akkulturation und bürgerliche Horizonterweiterung für notwendig und wünschenswert hielt.

Zu einer Idealisierung ländlicher Verhältnisse neigten hingegen die Erzähler des späten 19. und frühen 20. Jahrhunderts, nicht zuletzt deswegen, weil sie mit antijüdischem Ressentiment in der Regel ein modern-urbanes Phänomen verbanden (Ausnahme: Hessen); denn gegen das spätere Bollwerk eines salonfähigen, politischen Antisemitismus nahm sich der latent-christliche Judenhass auf dem Land wie Dorfspott aus (Jakob Loewenberg). Besonders die Autoren des konservativ-orthodoxen Lagers (Seligmann Meyer, Arthur Kahn, Pinchas Kohn) plädierten für einen Verbleib in den dörflichen Kehillot, deren Traditionsreichtum sich durchaus mit dem osteuropäischer Gemeinden messen konnte: nicht nur Thoragelehrsamkeit war auf deutschem Boden beheimatet, auch kabbalistisch beschlagene Rabbiner hatten bis weit ins 19. Jahrhundert dort gewirkt - ein gewichtiges Argument in der Diskussion um eine "jüdische Renaissance«. An dieses weitgehend vergessene Erbe galt es anzuknüpfen, daraus hieß es, sich zu erneuern: Der rasante Schrumpfungsprozess der ländlichen Gemeinden sollte durch »Wohlfahrtspflege« zunächst aufgehalten, aber auch umgekehrt werden. Das Land war attraktiv, weil es »regenerierend « auf Körper und Geist wirken konnte. Mit der erwarteten Rückkehr zu einem religiös observanten Leben verband sich die Hoffnung auf eine berufliche Neuorientierung der alten und neuen Dorfjuden, die sich durch den Betreib von Landwirtschaft (Arthur Kahn) vervollkommnen sollten: als Juden und als Deutsche.

Die Existenz eines jüdischen Bauernstandes sollte nicht nur den ultimativen Nachweis gelungener Integration in die deutsche Gesellschaft erbringen, sondern endlich den Schritt zu einem »naturgemäßen« Leben vollziehen. Während orthodoxe Schriftsteller sich von der Aufnahme landwirtschaftlicher Tätigkeit ein Erstarken religiöser Observanz versprachen, war Jahrzehnte zuvor in reformnahen Kreisen die Hinwendung zur Natur gerade mit einem Abrücken von den Kehillot verbunden gewesen. Das traditionelle Leben in den dörflichen Gemeinden war, so Krämer, zu einem guten Teil durch eine Skepsis gegenüber der 
unmittelbaren Umgebung bestimmt gewesen, wie man auch die Erzeugnisse, mit denen gehandelt wurde, nicht als Gaben der Natur begriff. Wollte man aber ein religiös und persönlich erfülltes, eben nicht von »irrigen Anschauungen « und Gemeinschaftsinteressen geleitetes Leben führen, hatte man sich die »natürliche« Umwelt gemeinsam mit der menschlichen zu erschließen; dazu musste man physisch wie psychisch heraus aus den drückenden eigenen vier Wänden. Bei Phöbus Philippson, wie später bei Loewenberg, stellt sich der rechte Glaube erst durch die Erfahrung des Erhabenen in der Natur ein und gewinnt die angestammte Religion an Innigkeit. Notwendige Voraussetzung dafür ist die Wahrung einer Distanz zur nächstgelegenen jüdischen Gemeinde, zu deren unmittelbarem Einzugsbereich man bewusst nicht gehören möchte. Denn der Begriff der Tradition war zu sehr mit Dünkel und Aberglauben verbunden, als dass sich eine freie kindliche Entfaltung in Interaktion mit der unmittelbaren Umgebung hätte ergeben können.

Ein Bild der Naturwidrigkeit und Trostlosigkeit landjüdischer Existenz vermitteln um die Jahrhundertwende zwei protoexpressionistische Werke, die den konfessionell-volksnahen Schriften jüdischer Autorinnen und Autoren fernstehen. Der aus einem traditionellen Haushalt stammende, doch weitgehend ohne religiöse Erziehung aufgewachsene Jakob Wassermann hatte wie Gustav Landauer, Spross einer gutbürgerlich-assimilierten Familie, seine Verbindung zum jüdischen Gemeinwesen früh gekappt. Beide fühlten sie sich der Lebensphilosophie Friedrich Nietzsches verpflichtet und lehnten jegliche Form institutionalisierter Religion als Werkzeug menschlicher Unterdrückung ab. Das landjüdische Milieu erscheint in beider Schriften als Herkunftsort im familiären wie historischen Sinn, als Wiege materialistischer Gesinnung und kleinbürgerlichen Erwerbsdenkens sowie als Reservoir niedriger kreatürlicher Instinkte. Traditionen würden aus Pflichtschuld und gesellschaftlichem Kalkül aufrechterhalten, niemals reflektiert und taugten kaum als lebensanschaulicher Anker eines freudlosen Daseins. Das »ewige« Judentum, wie es die Landjuden repräsentierten, zeichne sich durch religiöse Indifferenz aus, die das Mäntelchen der Tradition nur unzureichend decke; bei Landauer lässt es sich nur durch Tilgung ausmerzen oder durch »Konversion « zu einer universal-menschlichen Ordnung beheben. In Arnold Himmelheber vollzieht die schöne Judith diesen Schritt, indem sie eine Verbindung mit einem Nicht-Juden, ihrer Jugendliebe, eingeht, die der Forderung nach »naturgemäßer Zucht « nachkommt. Ist auch die Vorstellung, der Weg zur Natur führe von der jüdischen Gemeinschaft weg, in gewisser Weise dem aufklärerischen Geist jüdischer Reform geschuldet, haben sich doch die Begriffe semantisch zugespitzt: »Natur « ist nicht mehr (nur) ein spiritueller Erlebensraum, 
sondern auch ein Auftrag im »hygienischen« Sinn, den Individuum und Menschheit zu erfüllen hätten.

Wassermanns Roman Die Juden von Zirndorf kennt aber noch eine weitere Spielart des »Dorfjuden«: nämlich den weisen, weltfrommen, aber unerhörten Propheten, der ein bescheidenes Dasein führt und in der Landbevölkerung auf Wertschätzung stößt. Anders als bei Weill und Auerbach findet dieser menschenfreundliche, unbeirrbare Jude ein tragisches Ende, weil er an der Lieblosigkeit seiner Zeit zugrunde geht - ein Auslaufmodell, während der wahre Held des Romans sich von seinem Judentum emanzipiert und ein Dasein als »Übermensch» führt.

Mit dem Ausbau des »Dorfjuden « zum literarischen Ideenträger gerät zusehends die ländliche Gemeinde zur Projektionsfläche imaginierter Vergangenheiten und zum Experimentierfeld gesellschaftlicher Neuordnung. Ähnlich dem Dorf der "Dorfgeschichte« erfüllt die dörfliche Kehilla unterschiedliche Funktionen: sie steht für eine Vorstufe jüdischen Zusammenhalts im urbanen Raum, dient aber gleichzeitig als Abbild jüdischer Gesellschaft im Kleinen und Rückzugsgebiet frommer Sühner. »Authentische« Heimat ist sie dann, wenn sie das Individuum mit Grundvertrauen und einem Gefühl lebenslanger Zugehörigkeit auszustatten weiß (Jakob Loewenberg), aber auch, wenn sie sich durch einen lebendigen Minhag als altehrwürdig-jüdischer Boden legitimiert - fernab städtischer Musealisierung und Sentimentalisierung (Pinchas Kohn). Als Ende des 19., Anfang des 20. Jahrhunderts die deutsche »Heimat" den Juden wieder abgesprochen wird, schrumpft diese in der Erinnerung auf die Kindheitstage im Dorf zusammen, die man nicht selbst dort verbracht haben musste, um sich bei dem Gedanken daran unbeschwert oder schamhaft berührt zu fühlen. Und nicht zuletzt ließ sich das Vermächtnis der dörflichen Kleingemeinden auch andernorts antreten: in den bedeutend größeren städtischen Kehillot, wo Bevölkerungsstärke und staatlich bestellte Ordnungshüter wichtige Faktoren bei der Abwehr von Antisemitismus ausmachten (Salomon Luß) - oder im Naturreservat des eigenen Gartens (Jakob Loewenberg, Jakob Wassermann). 